\title{
Research on Real Surface Stability of Cone Bit Sliding Bearing Under Repeated Contact Condition
}

\author{
Wanzhong Li ${ }^{1, *}$, Chong Deng ${ }^{1}$, Yingqiang $\mathrm{Xu}^{2}$, Jian $\mathrm{Sun}^{3}$, Yazhou Feng ${ }^{1}$ and Duanyin Zhu ${ }^{1}$ \\ ${ }^{1}$ School of Mechanical Engineering, Xi'an Shiyou University, Xi'an, Shaanxi, P.R. China \\ ${ }^{2}$ School of Mechanical Engineering, Northwestern Polytechnical University, Xi'an, Shaanxi, P.R. \\ China \\ ${ }^{3}$ College of Mechanical and Electrical Engineering, Xi'an Polytechnic University, Xi'an, Shaanxi, P.R. \\ China
}

\begin{abstract}
The cone bit is the most principal rock breaking tool in drilling engineering. The statistical analysis of field data shows that the early damage and failure of sliding bearing is one of the main reasons that affect the service life and performance of the cone bit in the real drilling process. Aiming at the complex working environment of cone bit sliding bearing with heavy load, high temperature and mixed lubrication, the paper establishes a multilevel line contact model of rough surface and carries out the finite element modeling and analysis from the perspective of contact mechanics. Then the Von Mises stress, Residual normal deformation and Inelastic dissipated energy are obtained under the different cycles of loading-unloading. The results show that with the increase of cycles of loading-unloading, the part of edge area of the line contact enters into elastic shakedown and the asperities in the central area occur irreversible plastic deformation, which has a significant influence on the stability of the sliding bearing of the cone bit.
\end{abstract}

\section{Introduction}

The cone bit, shown in Fig.1, is the most principal rock breaking tool in drilling engineering. It has the effect of impacting, crushing and shearing formation rocks when working [1] and can adapt to formations with different hardness. It has irreplaceable advantages of PDC bit and diamond bit especially in deep wells and ultra-deep wells and occupies about $50 \%$ of the market in oil bits. In recent years, with the deepening of drilling footage, the service life of cone bit has been subjected to a severe challenge that the performance of cone bit directly affects the drilling quality, drilling efficiency and drilling cost [2]. The sliding bearing, a key component of cone bit, is a bridge to transmits torque and motion in the drilling process [3].

\footnotetext{
* Corresponding author: liwanzhong@xsyu.edu.cn
} 


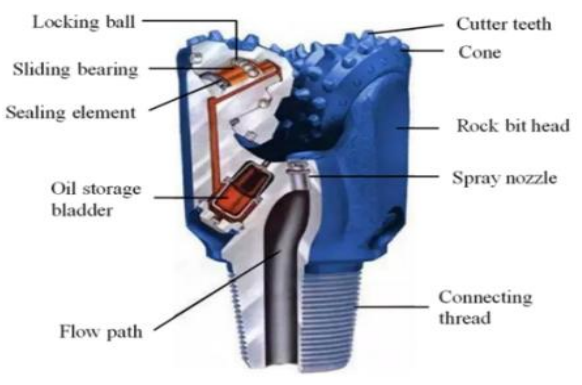

Fig. 1. Cone bit structure diagram.

Due to the limitation of drilling technology, wellhole size and wellbore structure, the sliding bearing of cone bit is characterized by small structure size, large load, low speed and poor lubrication conditions compared with ordinary bearing, which is one of the weaknesses in its structure. It is specifically manifested in four aspects that structural particularity, load particularity, relative speed particularity and working environment particularity [4].

According to the investigation on the quality problems of 64 cone bits of a certain specification [5], it is found that 46 cone bits were wear failures of bearing friction pairs joint surface, accounting for $71.9 \%$ of the total, which is much higher than broken and lost teeth, sealing failure, nozzle piercing and so on. Based on the anatomic analysis of the failed sliding bearing of cone bit, the main failure modes of the bearings include fracture failure, pitting and spalling of contact surface and wear failure [6].

The early damage and failure of sliding bearing is one of the main reasons affecting the service life and performance of the cone bit in the real drilling process [7]. Therefore, how to improve the wear resistance and stability of sliding bearing friction pair of cone bit and prolong its service life has become an urgent problem to be solved. The surface stability mainly includes the elastic-plastic deformation of asperities, friction and wear performance, reliability and lubrication performance, etc. The paper analyzes the repeated contact behavior of bearing real rough contact interface from the perspective of contact mechanics and explores the multi-scale repeated contact characteristics and friction and wear performance of sliding bearing contact interface under different working conditions. It provides theoretical and scientific basis for improving the operation stability and reliability of the bearing components.

\section{Theoretical model}

The contact surface of sliding bearing friction pairs has a certain roughness at microscopic scale and the contact on surfaces is actually the contact of asperities. The contact behavior of rough surface plays a vital role in the real bearing capacity, friction and wear performance of the sliding bearing.

From the analysis of macroscopic contact mechanics, the Hertz contact theory [8] regards the contact pair as the contact between two ideal smooth elastic curved surfaces. When the contact surface of the two components is cylindrical, line contact occurs on the contact surface and the contact pressure is distributed in a rectangular area [9]. At present, many analytical loading models have been proposed in the contact study of micro-rough surfaces, including $G W$ model [10], $C E B$ model [11], ZMC model [12] and $K E$ model [13]. When unloading, the deformation of the asperity can be divided into three cases that complete elastic deformation, elastic-plastic deformation and complete plastic deformation [14]. 
The micro contact multilevel model uses the overall level and asperity level to describe the rough surface profile [15]. According to the equal load on a single asperity under the overall level and asperity level, the contact models under the two levels can be connected. The contact between cone shaft hole and claw journal of cone bit sliding bearing is a complex linear contact. Therefore, the idea of multilevel contact model [16] can be applied to the line contact.

The line contact model of two random curved surfaces considering roughness can be equivalent to the line contact between a rough flat surface and an ideal smooth cylindrical surface $[10,17]$ shown in Fig.2 Using the idea of calculus, the line contact area with contact width of $2 \mathrm{a}$ is regarded as a set of discrete lines that parallel to the cylindrical generatrix direction ( $y$-axis). In the direction of contact width ( $x$-axis), the distance between the average line of asperity height and the rigid smooth cylindrical surface varies along the direction of contact width. The real contact area and load on each discrete line are solved by the idea of multilevel contact model and the real total contact area and contact load of line contact are obtained through integration method.

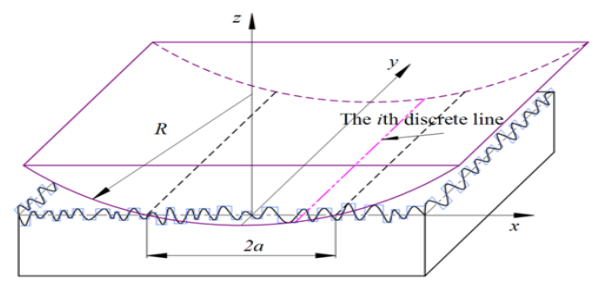

Fig. 2. Multilevel model applied to the line contact

According to the idea of the multilevel contact model, the bearing load on each asperity at overall level is calculated at first and then the real contact area and normal deformation of each asperity are calculated by using the contact model at asperity level. The contact area and load of asperity on each discrete line are related to the probability density distribution functions of asperity heights when load is applied. There is

$$
\left\{\begin{array}{l}
A_{i}=\eta A_{n} \int_{d_{s}}^{\infty} A\left(z-d_{s}\right) \psi(z) d z \\
F_{i}=\eta A_{n} \int_{d_{s}}^{\infty} f\left(z-d_{s}\right) \psi(z) d z
\end{array}\right.
$$

In the formula:

$A_{i}, F_{i}$-The real contact area and bearing load of asperity on the $i$ th discrete line in line contact area; $\eta$-Density of asperity; $A_{n}$-The apparent area of each discrete line obtained by Hertz theory; $d_{s}$-The distance between the rigid smooth flat surface and the average line of asperity height; $A\left(z-d_{s}\right), f\left(z-d_{s}\right)$-The real contact area and bearing load of single asperity related to the probability density distribution functions of asperity heights respectively.

The real total contact area and total contact load of line contact can be obtained by integral operation of discrete lines in the direction of contact width, that is

$$
A_{t o t}=\int_{-a}^{a} A_{i} d x, F_{t o t}=\int_{-a}^{a} F_{i} d x
$$

\section{Finite element simulation model}

The rough flat surface specimen is made of $12 \mathrm{Cr} 2 \mathrm{Ni} 4 \mathrm{~A}$ alloy structural steel for aeronautic gear. The $C r W M n$ used for the material of cylindrical specimen is the most common highcarbon alloy tool steel for making molds. In order to compare with the results of line 
contact model, the same material sample is used for the multilevel model of point contact. The $C r W M n$ alloy tool steel is also used for the sphere with the radius $R=5 \mathrm{~mm}$. See Table 1 for the relevant material performance indexes and geometric dimensions [18-20].

Table 1. Mechanical and surface properties of contact materials

\begin{tabular}{|c|c|c|}
\hline Material & $12 C r 2 N i 4 A$ & $C r W M n$ \\
\hline Elastic modulus $E[\mathrm{GPa}]$ & 209 & 209 \\
\hline Poisson ratio $v$ & 0.3 & 0.3 \\
\hline Profile arithmetic deviation $R a[\mu \mathrm{m}]$ & 0.24 & 0.10 \\
\hline Root mean square deviation of surface profile height $\sigma[\mu \mathrm{m}]$ & 0.31 & 0.13 \\
\hline Bulk curvature radius $R[\mathrm{~mm}]$ & $\infty$ & 5 \\
\hline Contact length $l[\mathrm{~mm}]$ & 12 & 12 \\
\hline Size $[\mathrm{mm}]$ & $24 \times 12 \times 24$ & $\Phi 10 \times 12$ \\
\hline Yield strength $S y[\mathrm{MPa}]$ & 910 & 2668 \\
\hline Hardness $(H=2.8 Y)[\mathrm{MPa}]$ & 2548 & 7470.4 \\
\hline
\end{tabular}

Using the method of Least mean square error approximation and combining with the contact surface profile height of the flat surface specimen measured by means of instrument, the parabolic approximation is performed on the profile in the direction of contact width and the results are shown in Fig.3.

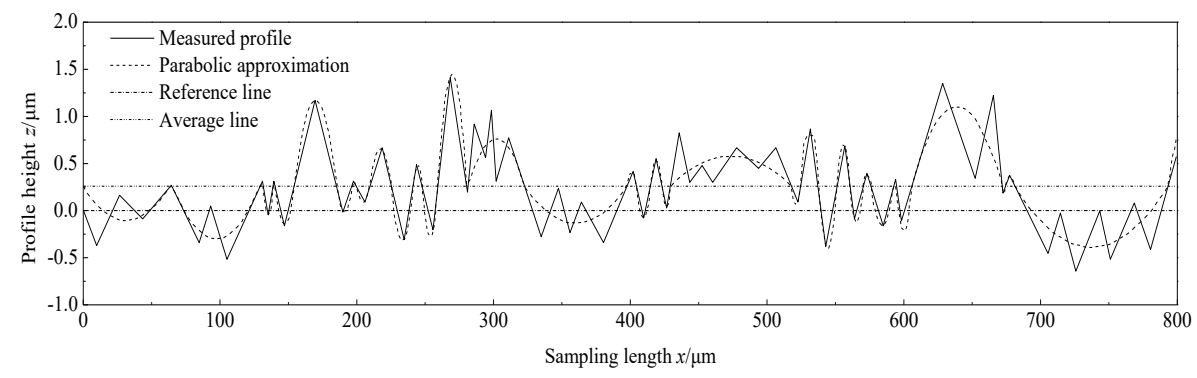

Fig. 3. Measured profile and parabolic approximation of the flat surface specimen

According to the distributions of profile heights and the topography approximation of rough surface, the lattice coordinates are imported into the finite element pre-processing software to generate the specimen surface which can be analyzed by the finite element, as shown in Fig.4(a). Taking into account the influence of computer truncation error on the calculation accuracy at microscopic scale, the length unit is $\mu m$, the time unit is $\mu s$ and the mass unit is $n g$ in the finite element modle. In the corresponding derived unit system, the force unit is $\mu N$, the stress unit is $M P a$ and the energy unit is $p J$. In order to simplify the calculation, the finite element modeling and calculation are mainly performed on the contact area of the flat surface specimen. 


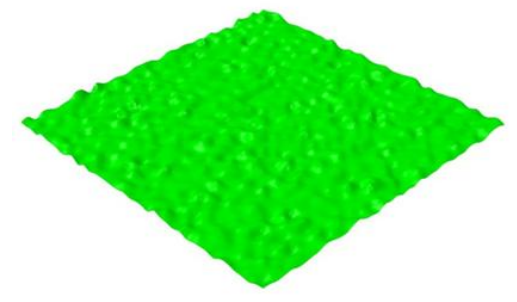

(a) Surface creation

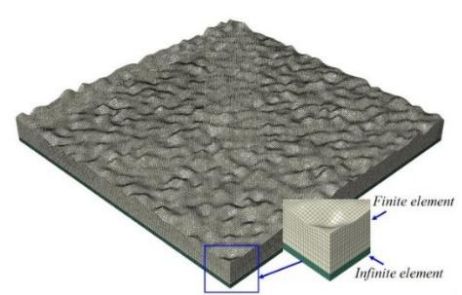

(b) Finite element model

Fig. 4. 3D finite element model of specimen

As shown in Fig.4(b), the area of model is $60 \mu m \times 60 \mu m$ and the thickness is $6 \mu m$. The model element is divided into two parts that the upper part using the traditional finite element $C 3 D 8 R$ with reduced integration and the lower part using the infinite element $C I N 3 D 8$. The model introduces an infinite element at the bottom of the specimen to replace the modeling method of applying the displacement boundary directly at the bottom of the specimen, which can reduce the stiffness modeling error of the specimen in the thickness direction.

As shown in Fig.5, there is a $3 D$ finite element contact model applied to line contact. The cylindrical specimen of the model is simplified into a rigid body and a contact relationship is established between the cylindrical specimens and the flat surface specimen, And the tangential contact behavior is defined as the friction-free. The Yield criterion of isotropic strengthening is adopted in the calculation and the yield behavior of the specimen material is considered. The circulation of load is controlled by the triangular waveform amplitude curve and subjected to the cyclic loading-unloading of 10 times.

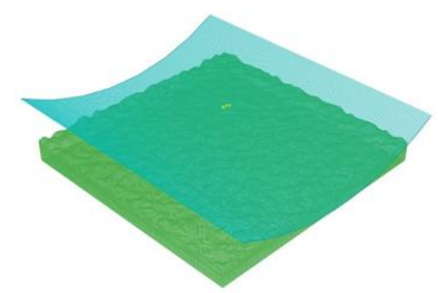

Fig. 5. 3D finite element contact model applied to line contact

\section{Results and discussion}

\subsection{The distribution of contact pressure}

During the loading of line contact, the maximum contact pressure occurs at the center of contact width and then decreases gradually to both sides until the edge of contact width where the contact pressure becomes zero.

\subsection{Von Mises stress}

The Fig. 6 shows the results of finite element simulation of Von Mises stress after the line contact flat surface specimen is unloaded when the cycles of loading-unloading $C_{n}=1,4,7,10$. It can be seen that the area where plastic deformation occurs is getting larger and larger 
after unloading, especially in the early stage of cyclic loading and with the increase of the cycles of loading-unloading, the area where plastic deformation tends to be stable gradually.

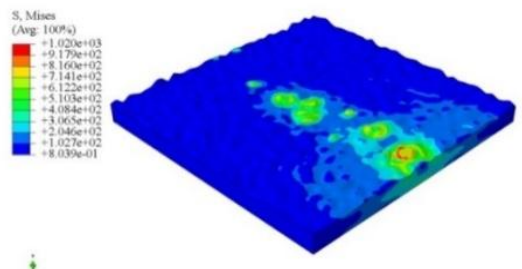

(a) $C_{n}=1$

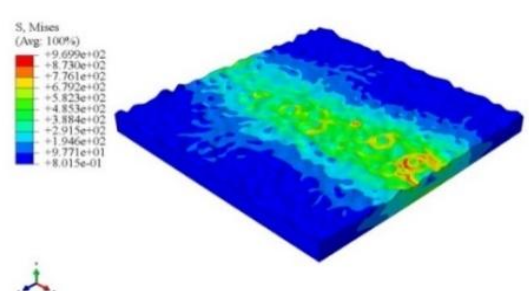

(c) $C_{n}=7$

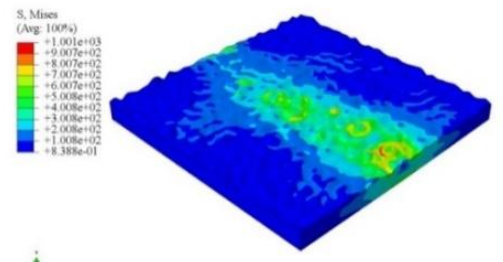

(b) $C_{n}=4$

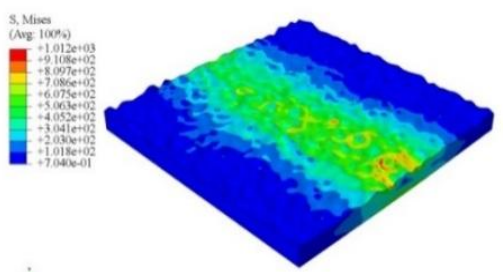

L.

(d) $C_{n}=10$

Fig. 6. Mises stress under line contact

\subsection{Residual normal deformation}

The Fig.7 shows the results of finite element simulation of Residual normal deformation after the line contact flat surface specimen is unloaded when the cycles of loadingunloading $C_{n}=1,4,7,10$. It can be seen that the Residual normal deformation of the cylindrical surface and the rough flat surface contact center becomes larger and larger and spreads to both sides with the increase of the cycles of loading-unloading, especially in the early stage of cyclic loading. With the increase of the cycles of loading-unloading, the Residual normal deformation tends to be stable gradually.
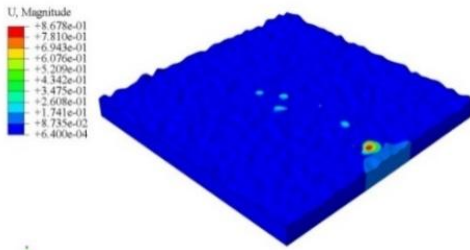

$\dot{L}$

(a) $C_{n}=1$
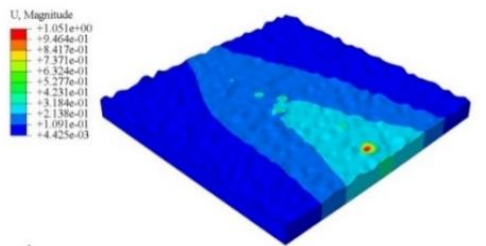

$\dot{L}$

(c) $C_{n}=7$

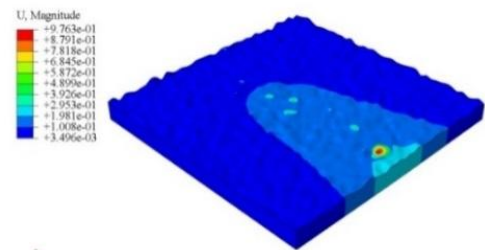

(b) $C_{n}=4$

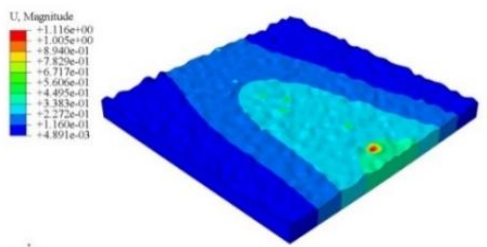

$\dot{1}$

(d) $C_{n}=10$

Fig. 7. Residual interference under line contact 


\subsection{Inelastic dissipated energy}

The Inelastic dissipated energy $(A L L P D)$ of line contact with different cycles of loadingunloading is extracted by $A B A Q U S$ software as shown in Fig.8(a). It can be seen that the Inelastic dissipated energy changes obviously in the early stage of cyclic loading-unloading and with the increase of the number of cycles, the change tends to be stable gradually. Except for generating a certain plastic deformation and appearing a residual stress distribution in the early stage, the surface of the specimen doesn't occur new plastic deformation anymore and it will be in a state of elastic shakedown.

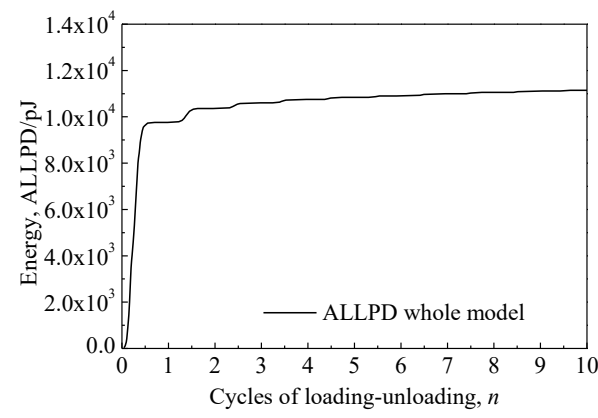

(a) Energy dissipated

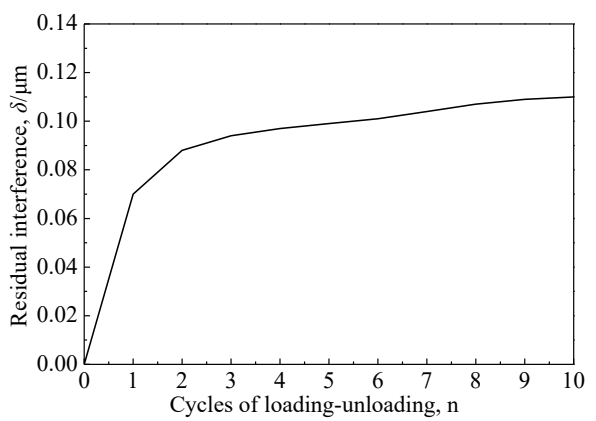

(b) Residual interference

Fig. 8. Simulation results of line contact

\section{Conclusions}

According to the complex working conditions of heavy load, high temperature, low speed and mixed lubrication of cone bit sliding bearing, a real multi-scale friction model between surface asperities and matrix is established to characterize the real topography evolution law of contact interface, which provides theoretical basis and experimental support for improving the service life of cone bit sliding bearing and further promote the application of bionic texture Tribology in the field of oil drilling and production equipment and tools.

(1) Completed the study of real surface topography evolution of cone bit sliding bearing, obtained the surface topography evolution results under different cycles of loadingunloading and explored the relationship between the plastic deformation area and the stability that larger plastic deformation area is, the worse the stability is and the easier the sliding bearing is to fail.

(2) It is found that the main factors affecting the contact surface stability of cone bit sliding bearing are plastic deformation area, residual normal deformation and inelastic dissipated energy. With the increase of cycles of loading-unloading, plastic deformation area, residual normal deformation and inelastic dissipated energy tend to be stable. Meanwhile, the contact surface of the sliding bearing also gradually stabilizes.

(3) With the increase of cycles of loading-unloading, the residual deformation is gradually stable after increasing to about $0.09 \mu \mathrm{m}$ and the energy dissipation is also in a stable state after increasing to about $1.1 \times 10^{4} \mathrm{pJ}$, which further verifies the stability of contact surface of cone bit sliding bearing after loading-unloading. 


\section{Acknowledgements}

The authors are truly grateful for the support by the Scientific Research Program Funded by Shannxi Provincial Education Department(Program No. 19JK0659) and Shaanxi Provincial Natural Science Basic Research Project(Program No. 2021JQ-606)

\section{References}

[1] L. Zhong, G. Wei, Y. Li, et al. Experimental study on the effect of surface texture shape on the tribological properties of cone bit bearing. J. Lubrication and sealing, 45 (3): 27-32(2020)

[2] X.H. Xiao, S. Chen, T. Guo, et al. Effect of ceramic film on friction and wear properties of cone bit sliding bearing. J. Metal heat treatment, 41 (10): 75-79(2016)

[3] X He, M.Y. Li, G.R. Wang, et al. Comparative analysis of three processing methods for surface texture of cone bit bearing. J. Surface technology, 47 (3): 28-35(2018)

[4] K.S. Wu, D.K. Ma. Particularity and main research direction of cone bit sliding bearing. J. Petroleum machinery, 29 (4): 52-54(2001)

[5] Y. Wang. Improved design of lubrication device for cone bit. J. Mining technology, 11 (6): 80-81(2011)

[6] Z.Q. Huang, X.F. Wang, X.F. Tu, et al. Failure analysis of sliding bearing of three cone bit. J. Journal of Southwest Petroleum University (Natural Science Edition), 30 (3): 136-138(2008)

[7] H.Q. Xiao, S.H. Liu, Y. Chen, et al. Tribological properties of four bearing steels in water-based mud. J. Journal of mechanical engineering, 54 (13): 153-158(2018)

[8] H. Hertz. On the contact of elastic solids. J. Reine and angewandte Mathematik, 94: 156-171(1882)

[9] B. Bhushan. Introduction to tribology. New York: John Wiley\&Sons, (2002)

[10] J.A. Greenwood, J.B.P. Williamson. Contact of nominally flat surfaces. Proceedings of the Royal Society of London Series-A, 295(1442): 300-319(1966)

[11] W.R. Chang, I. Etsion, D.B. Bogy. An elastic-plastic model for the contact of rough surfaces. Journal of Tribology-Transactions of the ASME, 109(2): 257-263(1987)

[12] Y.W. Zhao, D.M. Maietta, L. Chang. An asperity microcontact model incorporating the transition from elastic deformation to fully plastic flow. Journal of TribologyTransactions of the ASME, 122(1): 86-93(2000)

[13]L. Kogut, I. Etsion. Elastic-plastic contact analysis of a sphere and a rigid flat. Journal of Applied Mechanics-Transactions of the ASME, 69(5): 657-662(2002)

[14] Y. Kadin, Y. Kligerman, I. Etsion. Unloading an elastic-plastic contact of rough surfaces. Journal of the Mechanics and Physics of Solids, 54(12): 2652-2674(2006)

[15]A. Beheshti, M.M. Khonsari. Asperity micro-contact models as applied to the deformation of rough line contact. Tribology International, 52(8): 61-74(2012)

[16]F.S. Wang, J.M. Block, W.W. Chen, et al. A multilevel model for elastic-plastic contact between a sphere and a flat rough surface. Journal of Tribology, 131(2): 021409(2009)

[17] J.A. Greenwood, J.H. Tripp. The Elastic contact of rough spheres. Journal of Applied Mechanics, 34(1): 153-159(1967) 
[18]Y.Q. Xu, G.Z. Lv. Research on the prediction method of contact fatigue crack initiation life of aviation gear. Journal of Northwestern Polytechnical University, 21(4): 473-476(2003)

[19]X.L. Liu, W.Y. Zhang. Selection and Application of Cold Work Die Steel. Forging Technology, 32(6): 13-17(2007)

[20] W.Z. Li, Y.Q. Xu, J. Sun, et al. Elastic-plastic loading and unloading multilevel contact model for rough surfaces. China Surface Engineering, 28(4): 39-46(2015) 